\title{
Redes de Ingeniería
}

http://revistas.udistrital.edu.co/ojs/index.php/redes/index

R $\sum$ DES DE INGENIERÍA

[Rompiendo las barreras del conocimiento]

http://dx.doi.org/10.14483/udistrital.jour.redes.2016.2.a06

ARTíCULO DE INVESTIGACIÓN CIENTÍFICA

\section{Impacto de la fragmentación espacial sobre el valor de los inmuebles de uso residencial utilizando la metodología de precios hedónicos, caso de estudio: Mosquera, Cundinamarca}

\section{Impact of spatial fragmentation on value of residential housing using the hedonic price methodology, case of study: Mosquera, Cundinamarca}

\author{
Laura Angélica Cadena Contreras ${ }^{1}$ Wendy Yesenia Hernández Suárez² \\ Héctor Javier Fuentes López ${ }^{3}$
}

Para citar este artículo: Cadena L.A., Hernández W.Y. y Fuentes H.J.(2016). Impacto de la fragmentación espacial sobre el valor de los inmuebles de uso residencial utilizando la metodología de precios hedónicos, caso de estudio: Mosquera, Cundinamarca. Revista Redes de Ingeniería. 7(2), 170-182. Doi: 10.14483/udistrital.jour. redes.2016.2.a06

Recibido: 25-abril-2016 / Aprobado: 20-junio-2016

\section{Resumen}

Este estudio determina el impacto económico de la fragmentación espacial sobre el valor comercial de los inmuebles de uso residencial utilizando el método de precios hedónicos. Se plantea un modelo basado en los atributos que se consideran más relevantes, teniendo en cuenta que este es un bien heterogéneo que se encuentra en función de sus características físicas y su entorno. La metodología es aplicada para el análisis del mercado inmobiliario del municipio de Mosquera, Cundinamarca. Los resultados de los estimadores econométricos espaciales sugieren que la fragmentación, además de ser significativa, si se presenta en bajos niveles implica un impacto negativo sobre el valor comercial de los inmuebles, mientras que si lo hace en altos niveles produce un incremento en el mismo.

Palabras clave: econometría espacial, fragmentación espacial, modelo hedónico, usos de suelo.

\begin{abstract}
This study develops the methodology to determine the economic impact of spatial fragmentation on the commercial value of property with residential use, it is using the hedonic price method based on attributes that are considered most relevant for the model, it is considering that this is an heterogeneous property that is in function on their physical characteristics and their environment. The methodology is applied to analyze the real states values in the municipality of Mosquera, Cundinamarca. The results of spatial econometric estimates suggest that fragmentation besides being significant, at low levels it impacts negatively the residential house prices and at high levels it increases the price.
\end{abstract}

Keywords: hedonic model, land uses, spatial econometric, spatial fragmentation.

1. Estudiante de Ingeniería Catastral y Geodesia, Universidad Distrital Francisco José de Caldas. Correo electrónico: lacadenacontreras@gmail.com

2. Estudiante de Ingeniería Catastral y Geodesia, Universidad Distrital Francisco José de Caldas. Correo electrónico: wyhernandezs@gmail.com

3. Profesor, Universidad Distrital Francisco José de Caldas. Correo electrónico: hjavierf@gmail.com 


\section{INTRODUCCIÓN}

Como resultado de la reducida intervención estatal, los inversionistas y promotores inmobiliarios han desarrollado una dinámica en la que el crecimiento urbano depende de la relación entre la oferta y la demanda y, en consecuencia, se ha producido una transformación morfológica y funcional del territorio [1]. Los antecedentes indican que para modelar el valor que se le da a este tipo de bienes se requiere un proceso más complejo al utilizado habitualmente para atender las demandas del consumidor, ya que es necesario considerar las características tanto físicas como de localización.

El artículo se enfoca en determinar el impacto que genera la presencia de la fragmentación espacial sobre los inmuebles de uso residencial. De acuerdo a Altinok y Cengiz [2] la discordancia de las actividades del uso de la tierra y de las propiedades físicas del espacio se conoce como fragmentación espacial; un alto grado de este tipo de fragmentación demuestra la falta de integración del paisaje, siendo dividido en varias áreas (o fragmentos), cada una con diferente actividad del uso de la tierra, como residencial, industrial, etc. [3].

Dado el objetivo del estudio, se requiere analizar las características asociadas a la presencia de la fragmentación espacial reflejadas en el valor de las propiedades residenciales, por lo que es necesario indagar sobre los aportes existentes. Koster y Rouwendal [4] presentan el análisis de la influencia que tiene la pluralidad de los usos de la tierra en los valores inmobiliarios residenciales ubicados en Rotterdam (Holanda), este menciona la existencia de tipos de uso del suelo que afectan positivamente los precios de la vivienda.

Los efectos implícitos del precio pueden ser estimados a través de un análisis de precios hedónicos [5]; aunque este tipo de análisis se ha usado frecuentemente para examinar los efectos de actividades específicas del uso de la tierra sobre el precio de los bienes, son reducidas las investigaciones empíricas enfocadas a la fragmentación espacial. Kockelman [6] involucra no solamente los valores comerciales sino también los de renta de las casas localizadas en San Francisco, California, encontrando una relación estadísticamente significativa y negativa entre los valores de las propiedades y la fragmentación espacial; resultados similares a los obtenidos por Kuethe [3] en su estudio realizado en la ciudad de Milwaukee, Wisconsin. Por otro lado, Geoghegan, Wainger y Bockstael [7] indican en su estudio, que la fragmentación espacial no tiene impacto en los valores de los inmuebles.

Dadas las ambigüedades de las conclusiones obtenidas en los estudios previos, el presente artículo busca contribuir a la literatura existente, además de ser la base para investigaciones posteriores como herramienta para analizar la problemática que viven los municipios del país, debido al conflicto de usos del suelo y todos aquellos temas que implican el ordenamiento territorial, como la falta de planeación y gestión urbana, puesto que actualmente pasó de ser una fortaleza a una debilidad [8].

El contenido del artículo se encuentra estructurado de la siguiente manera, después de la introducción, en la segunda sección son descritas la estructura general del método de precios hedónicos y las consideraciones necesarias para abordarse desde la perspectiva de la econometría espacial, en la tercera sección se presenta el estudio de caso para el municipio de Mosquera, con sus respectivos resultados. Finalmente, en las dos últimas secciones se aportan discusiones y conclusiones fundamentadas en estimaciones obtenidas, manifestando las ventajas de estas técnicas en el tratamiento de datos.

\section{EL MÉTODO DE PRECIOS HEDÓNICOS}

El modelo teórico en el que se basa el estudio de los precios hedónicos fue desarrollado por Rosen [5], quien plantea que los bienes son valorados por 
la utilidad que brindan sus atributos; los precios y las características asociadas a cada uno de estos bienes definen un conjunto de precios implícitos o "hedónicos". Esta teoría es formulada bajo el supuesto de un mercado en equilibrio, en el que los ofertantes y demandantes compiten por encontrar las mejores características en el bien deseado.

La teoría de precios hedónicos define el suelo y los inmuebles como un bien compuesto descrito por un conjunto de características físicas, de localización y entorno, identificadas por la ecuación de regresión:

$$
Y=\mathrm{X} \beta+\varepsilon
$$

Donde $Y$ representa el vector $N \times 1$ del valor comercial de los inmuebles. X es la matriz de dimensiones $N \times K$, siendo $K$ las diferentes características que se definen como variables económicas homogéneas, a partir de las cuales se construyen los bienes heterogéneos. La contribución de cada atributo para el valor de un inmueble es capturada por el vector $\beta$ de $K \times 1$, el cual señala la variación parcial de $Y$ con relación a la variación de $\mathrm{X}$. Donde el término del error que asume la forma de una distribución normal con media cero y varianza constante $\varepsilon \sim N\left(0, \sigma^{2}\right)$. Entre los atributos comúnmente considerados se encuentran las características físicas de la construcción y aquellos relacionados con el entorno en el que el predio se halla ubicado [9].

La teoría clásica de localización sugiere que los precios de la vivienda disminuyen a medida que la distancia al centro urbano aumenta, por esta razón la ubicación es frecuentemente modelada a través de una variable que determine esta distancia; sin embargo, el modelo monocéntrico tradicional no logra describir acertadamente la estructura espacial de los múltiples núcleos que se encuentran a lo largo de las áreas urbanas modernas [10]. Por otro lado, estudios como el de McMillen [11] se orientan a demostrar que la desaparición del modelo clásico es exagerada, puesto que la ciudad central sigue dominando los patrones espaciales urbanos y las ideas básicas del modelo se aplican a las ciudades policéntricas más complejas. El modelo sigue siendo un instrumento analítico útil, puesto que gran parte de la aparente disminución de la capacidad explicativa del modelo de ciudad monocéntrica es en realidad una mala interpretación de la evidencia empírica.

Otras distancias usualmente consideradas en este tipo de metodología, son aquellas relacionadas a los sitios más representativos en cuanto a cultura, educación, servicios, comercio, industria y zonas verdes y espacios abiertos; ya que estas se utilizan para el cálculo de algunas variables que afectan directamente el valor comercial de los inmuebles.

\section{ESTIMACIÓN DEL MODELO}

Para la estimación del modelo de precios hedónicos se ha utilizado tradicionalmente el método de Mínimos Cuadrados Ordinarios (MCO); sin embargo, considerar la dimensión espacial implica preguntarse dónde ocurre el fenómeno y de qué manera dicha distribución geográfica condiciona o influye en estos. De ahí que los métodos de análisis espacial han adquirido mayor relevancia en estudios que requieren capturar de manera más precisa las interacciones entre las observaciones espaciales, puesto que emplean la ubicación tanto en términos absolutos (dónde ocurre), como en términos relativos (distribución y distancia entre las observaciones) [12].

El Análisis Exploratorio de Datos Espaciales (AEDE) es considerado como una herramienta para conocer la variación del valor comercial de los inmuebles, ya que permite analizar la presencia de heterogeneidad y dependencia espacial en un espacio geográfico. [13].

La heterogeneidad espacial hace referencia a la variación de las relaciones en el espacio. La dependencia o autocorrelación espacial se evidencia 
ante la presencia de una relación funcional entre lo que ocurre en un determinado punto del espacio y la dinámica que este tiene con otro lugar, es decir, en un proceso espacial lo que ocurre en una unidad, puede estar afectado por sus vecinos y al mismo tiempo los cambios en dicha unidad pueden afectar a estas vecindades [14].

Anselin [15] diferencia la existencia de dos tipos de dependencia espacial: la primera es considerada como ruido, porque no afecta los coeficientes del modelo y solo interesa para mejorar la estimación; esta se encuentra relacionada al término del error, razón por la cual se viola el supuesto de la no correlación entre errores que asume la regresión de mínimos cuadrados. Si se ignora esta dependencia los estimadores de la regresión serán ineficientes, aunque no sesgados [16]. La segunda hace referencia a la autocorrelación espacial sustantiva, que se presenta cuando la variable dependiente está determinada por el valor que asume en las unidades geográficas vecinas. En dado caso que, por el método de mínimos cuadrados, esta se llegara a ignorar, la estimación de los coeficientes será sesgada, lo que conlleva a tener problemas en la estimación y el signo de los coeficientes, la significancia de los mismos y la bondad del ajuste del modelo [12].

La presencia de la autocorrelación en el término del error puede ser tratada a través de un Modelo Espacial del Error (MEE), que tiene como supuesto que el origen de la dependencia espacial que contiene la variable endógena es el resultado de la autocorrelación del término del error y la distribución geográfica de las variables explicativas [17]. La estructura del MEE está dada por:

$$
\begin{gathered}
Y=\mathrm{X} \beta+u \\
u=\lambda \mathrm{W} u+\varepsilon
\end{gathered}
$$

Donde $u$ es el término de perturbación que se asume para seguir un proceso autoregresivo espacial, el cual está en función del parámetro espacial desconocido $\lambda$ y una matriz exógena de pesos espaciales $\mathrm{W}$ de $N \times N$. Esta matriz identifica la vecindad más representativa para cada observación, los elementos fuera de la diagonal toman valores más grandes que cero cuando dos observaciones comparten una relación significativa, de igual modo ninguna observación puede ser vecina de sí misma y, como resultado, los elementos de la diagonal son iguales a cero. El signo de $\lambda$ indica la naturaleza y grado de la dependencia espacial de los residuales de la regresión. El término $\varepsilon$ representa el vector de errores no correlacionados. Teniendo en cuenta que los supuestos de los MCO son violados, existen procedimientos alternos para la estimación que incluyen la máxima verosimilitud y momentos generales (GM) [3].

La alternativa para modelar este tipo de autocorrelación sustantiva es el modelo de Rezago Espacial, que considera la dependencia espacial introduciendo un rezago. Este tipo de modelo representa la dinámica entre la variable dependiente y las variables independientes en las unidades vecinas. [12]. El modelo de Rezago Espacial toma la forma:

$$
Y=\rho \mathrm{W}_{\mathrm{y}}+\mathrm{X} \beta+\varepsilon
$$

Siendo $\rho$ un parámetro desconocido de características espaciales, el signo de este parámetro indica la naturaleza de la dependencia espacial (positiva o negativa), sugiriendo así la existencia de la misma en los valores observados del mercado. El término del rezago espacial $\mathrm{W}_{\mathrm{y}}$, es endógeno, así los $\mathrm{MCO}$ pueden conducir a una estimación parcial de los coeficientes; sin embargo, no es necesario limitarse a este método, puesto que existen alternativas como la máxima verosimilitud y mínimos cuadrados en dos etapas [18].

Para la correcta especificación y aplicación de un método de estimación es necesario detectar y analizar el tipo de dependencia espacial que presenta el modelo que se desarrolla o con que se trabaja [19]; para su tratamiento el procedimiento más 
común es aplicar el Índice de Morán y la prueba del Multiplicador de Lagrange (LM) a los residuos de la regresión de MCO [20]. Se espera que estos residuos tengan autocorrelación en caso de que en el modelo la relación espacial no haya sido tenida en cuenta correctamente.

El Índice de Morán mide la tendencia de valores similares a agruparse en el espacio, estos valores varían entre - 1 y 1 , donde 0 implica la no existencia de un patrón definido, mientras valores tendientes a -1 indicarían autocorrelación negativa y 1 el máximo de autocorrelación positiva [12]. Para los LM se tiene que estos siguen una distribución $X^{2}$ asintótica y la hipótesis nula es que no existe dependencia espacial. De acuerdo con Anselin, Bera, Florax, y Yoon [21] para escoger el modelo más apropiado es necesario comparar los estadísticos $L M_{\lambda}$ y $L M_{\rho}$ : si $L M_{\lambda}$ es robusto y también más significativo que $L M_{\rho}$, sin ser este último robusto, el modelo debe ser especificado con la dependencia en el error; en el caso de que ambas condiciones se cumplan para $L M_{\rho}$ la correcta especificación es de dependencia sustancial.

Para estimar la ecuación hedónica es necesaria la elección de la forma funcional adecuada, la cual puede ser lineal o no. Debido a que no se ha establecido un criterio para la selección de una forma funcional que ofrezca mejores resultados, dicha elección se reduce a una cuestión empírica. Por esta razón, se contrastan diferentes formas funcionales y se utiliza la que proporcione un mejor ajuste. Pueden destacarse entre las formas funcionales más utilizadas tradicionalmente la lineal, la semilogarítmica y la doblemente logarítmica [22]. Bajo este supuesto, la forma funcional que mejor demuestra la relación entre el precio y las variables explicativas en el artículo es la semilogarítmica; además del buen ajuste que ofrece, de acuerdo con Selim [23] es la preferida porque la estimación de los coeficientes generados puede ser interpretada como la proporción del precio de un bien que es directamente atribuible a las características respectivas del mismo. Esta forma funcional es definida mediante la siguiente expresión:

$$
\ln Y=\mathrm{X} \beta+\varepsilon
$$

Con el ejemplo de Malpezzi [24] en un modelo lineal, el valor agregado de un tercer baño en una residencia de un dormitorio, es el mismo que se suma a una con cuatro dormitorios, lo que parece poco probable, contrastando con el modelo semilogarítmico, que permite variaciones en el valor monetario de una característica particular, el precio de un componente depende proporcionalmente de otras características de la vivienda como el tamaño y la calidad. Además, su interpretación es sencilla, puesto que un coeficiente puede ser interpretado como el porcentaje de variación aproximado en el precio, dado un cambio unitario en la variable independiente, lo que se conoce como "semielasticidades". Por ejemplo, si el resultado de las ponderaciones de una variable que representa a los garajes es 0,09 , entonces un garaje adicional incrementaría el valor de la vivienda en $9 \%$.

\section{Fragmentación}

Teniendo en cuenta que los objetivos del artículo se enfocan al análisis del impacto que tiene la fragmentación espacial sobre el valor del suelo en los predios de uso residencial, es necesario realizar un modelo que involucre no solo los valores comerciales y características físicas de los inmuebles, sino también la diversidad de las actividades del uso de la tierra.

El grado de fragmentación para cada unidad de estudio se calcula de la siguiente manera:

1. Es imprescindible disolver los linderos entre los que tienen una misma área de actividad del suelo; por ejemplo, si dos lotes consecutivos tienen un uso de distrito de adecuación de tierras el límite entre estos se remueve y el resultado es un parche del mismo uso. 
2. Una vez son identificados los respectivos parches, se procede a utilizar la herramienta Patch Analyst, ${ }^{4}$ para obtener las variables área y perímetro; y así adquirir la razón entre el perímetro y el radio del área (medida de la fragmentación espacial). Este radio indica si la fragmentación es pequeña o se extiende y fue previamente usado por Geoghegan, Wainger y Bockstael [7].

\section{CASO DE ESTUDIO}

La disminución de la intervención estatal en la gestión urbana ha permitido el aumento de la acción de agentes privados en el desarrollo territorial, que se ha materializado en las diferentes ofertas de proyectos de vivienda, generando así el fenómeno de fragmentación espacial en el territorio de los municipios aledaños a la capital, sobre los cuales ha tenido mayor influencia debido a su expansión [25].

Lo primero que se definió fue el área sobre la que se iba a realizar el estudio; debido a los resultados de una investigación a priori que tenía como propósito la búsqueda de los municipios que contaran con mayor nivel en su desarrollo territorial, el análisis se orientó hacia Mosquera, puesto que en el año 2015 fue clasificado por el "Departamento Nacional de Planeación", en el tercer puesto entre los municipios con mejores resultados en el desempeño integral de los 1102 que conforman el país y como el que mejor invirtió su presupuesto a nivel departamental en el momento del cumplimiento de las metas propuestas en el Plan de Desarrollo Territorial [26].

Después de definir donde se enfocaría el estudio, fue necesario buscar datos correspondientes al mercado inmobiliario de Mosquera, realizando tanto trabajo en campo como virtual con formatos electrónicos ofrecidos por diferentes agentes mediante páginas web; cabe aclarar que la búsqueda se limitó a las ofertas correspondientes al último trimestre del año 2015 y el primer bimestre del año 2016. Paralelo al trabajo realizado en campo, se procedió a adquirir información de tipo digital georeferenciada del Municipio, solicitada a la Secretaria de Planeación y Ordenamiento Territorial de Mosquera y entregada en medio magnético. Se realizó la revisión del PBOT (Plan Básico de Ordenamiento Territorial) apoyándose en los anexos del mismo, con lo que fue posible observar la clasificación y reglamentación de los usos allí existentes.

Con el trabajo de campo se identificaron los sitios más representativos de Mosquera en cuanto a cultura, educación, servicios, comercio, industria, centro del municipio y zonas verdes y espacios abiertos; ya que estos fueron necesarios para el cálculo de algunas variables que afectan directamente el valor comercial de los inmuebles.

Posteriormente, se relacionó la información georreferenciada con los datos correspondientes a las ofertas, es decir, se ubicó espacialmente cada oferta en el predio correspondiente. A fin de establecer la escala de trabajo deseada (a nivel de manzanas), se eliminaron los límites prediales; teniendo en cuenta que el propósito de la investigación se restringió al uso residencial (neto o con comercio y servicios) de carácter urbano, se filtraron las manzanas que cumplieran con estas características. Para consolidar la base de datos del proyecto se procedió a seleccionar únicamente las manzanas que presentaran una o varias ofertas, con lo que la muestra se ajustó a un total de 200 datos que incluyen el valor comercial del inmueble y un número de características de cada uno de los predios para usarlas como variables explicativas en el modelo de precios hedónicos. La Tabla 1 muestra la información utilizada para el modelo de precios hedónicos.

1. Patch Analyst es una extensión del software ArcGis, complemento que facilita el análisis espacial de los diferentes parches que se presentan en el paisaje, asignando a cada uno de estos sus respectivos atributos. [39] 
Tabla 1. Resumen de estadísticas: transacciones y datos del uso de la tierra 2015 - 2016 Mosquera, Cundinamarca.

\begin{tabular}{|c|c|c|}
\hline Variable & Media & Std. Dev \\
\hline Precio de venta (COP Pesos) & $\$ 227.435 .538$ & $\$ 112.966 .052$ \\
\hline Habitaciones & 3,7592 & 1,6363 \\
\hline Baños & 2,7317 & 1,0756 \\
\hline Edad (Años) & 6,0568 & 4,4082 \\
\hline Área construida $\left(\mathrm{m}^{2}\right)$ & 119,926 & 63,450 \\
\hline \multicolumn{3}{|l|}{ Distancia $(\mathrm{km})$} \\
\hline Centro del municipio & 1,3018 & 1,2168 \\
\hline Uso comercial & 1,2107 & 1,1007 \\
\hline Uso cultural & 0,9272 & 1,2576 \\
\hline Uso educativo & 0,7152 & 0,5678 \\
\hline Uso industrial & 0,8013 & 1,0838 \\
\hline Uso servicios y salud & 0,8835 & 0,5195 \\
\hline Zonas verdes y espacios abiertos & 0,2662 & 0,5065 \\
\hline Fragmentación & 0,1405 & 0,0879 \\
\hline \multicolumn{3}{|l|}{ Dummy (proporción \%) } \\
\hline Residuos (rellenos sanitarios) & 0 & \\
\hline Riesgos industriales & 0 & \\
\hline Riesgo de remoción & 0 & \\
\hline Garajes & 58 & \\
\hline
\end{tabular}

Para las variables tipo dummy correspondientes a los residuos, riesgos industriales y de remoción, teniendo en cuenta que su nivel de significancia es nulo, no se consideró relevante su inserción en el modelo; situación similar se presentó con la dummy garajes, en la que, dado su elevado nivel porcentual, se evidenció que la inclusión de la misma no generaría variaciones notables en el valor comercial del inmueble.

Teniendo la información de los puntos representativos anteriormente mencionados, se procedió a ubicarlos en la herramienta SIG, agrupándolos según su uso y el centro del municipio; por ser necesario para realizar el cálculo de las distancias entre las manzanas, el centro del municipio y cada una de estas áreas de actividad.

Teniendo en cuenta lo propuesto por McMillen [11] y Alonso [10] sobre el modelo de la teoría clásica de localización, se considera que la morfología espacial de Mosquera a pesar del desarrollo, ha mantenido un carácter monocéntrico, por lo que la expectativa es que el precio de los inmuebles sea mayor a medida que la oferta se ubique más cerca del centro.

En la Tabla 2 se disponen el número de parches y promedio de la fragmentación espacial (como medida calculada por la diferencia entre el perímetro y el radio del área) correspondiente a cada una de estas categorías. La Secretaria de Planeación del municipio realiza y define la clasificación del uso de la tierra en dos zonas, la primera es la urbana que contiene diecisiete áreas de actividad, la segunda es la rural, clasificada en cuatro tipos de suelo (protección, rural, suburbano y expansión) con un total de veinte áreas de actividad [27]. Con el propósito de optimizar el cálculo de la fragmentación, esta clasificación se delimitó en catorce áreas de actividad del uso del suelo para la totalidad del municipio (Tabla 2). 
Tabla 2. Resumen de las estadísticas: clasificación del uso de la tierra 2015-2016 Mosquera, Cundinamarca.

\begin{tabular}{lcc}
\hline & & Promedio \\
Áreas de actividad del uso del suelo & Cantidad & Perímetro / Radio del área \\
\hline Residencial neto & 280 & 0,0161 \\
Residencial con comercio y servicios & 127 & 0,0563 \\
Comercio y servicios & 59 & 0,0209 \\
Industrial & 12 & 0,0037 \\
Integral mixta & 12 & 0,0077 \\
Institucional & 15 & 0,0041 \\
Dotacional & 9 & 0,0203 \\
Centros poblados & 111 & 0,0313 \\
Urbanos básicos & 1 & 0,0510 \\
Plataformas logísticas & 27 & 0,0037 \\
Distrito de adecuación de tierras & 384 & 0,0026 \\
Minería & 76 & 0,0021 \\
Forestal Productora-Protectora & 76 & 0,0016 \\
Suelo de Protección & 19 & 0,0037 \\
\hline
\end{tabular}

Los resultados de la estimación semilogarítmica de precios hedónicos por el método MCO son presentados en la primera columna de la Tabla 3, en esta también se incluyen los del test de los multiplicadores de Lagrange para ambos modelos (Rezago Espacial y Error Espacial), al comparar estos estadísticos $\left(L M_{\lambda}\right.$ y $\left.L M_{\rho}\right)$ para seleccionar el modelo más adecuado, se evidenció que los dos son significativos, al igual que su robustez. Anselin, Bera, Florax y Yoon [21] sugieren que cuando ambos test son estadísticamente significativos, el de mayor valor será preferido, lo que conllevó a elegir el MEE, que como se mencionó anteriormente, mitiga los problemas asociados con la dependencia espacial omitida o las variables no observadas.

Como resultado, el modelo de precios hedónicos es estimado siguiendo la especificación del error espacial utilizando el estimador de Máxima Verosimilitud calculado en GeoDa. El software permite efectuar todas las etapas requeridas para el manejo de este tipo de datos; representación cartográfica, estudio analítico y gráfico de la autocorrelación espacial, análisis exploratorio multivariante y regresión espacial [28]. El resultado de la estimación del error espacial se presenta en la columna dos de la Tabla 3.

\section{DISCUSIÓN DE LOS RESULTADOS}

La Tabla 3 compara los resultados del MCO y el MEE, en esta se muestran los beneficios de incluir la autocorrelación en los errores, los que se evidencian en la estimación del error estándar y en la variación de los coeficientes. Visualizándose en el cambio de la significancia del coeficiente de baños, el cual aumenta en el MEE.

La mayoría de los coeficientes estimados de las especificaciones del error espacial son estadísticamente significativos. Las excepciones incluyen la edad, el uso industrial y el uso de servicios. 
Tabla 3. Resultados del modelo hedónico.

\begin{tabular}{|c|c|c|c|c|c|c|}
\hline \multirow[b]{2}{*}{ Variable } & \multicolumn{2}{|c|}{$\begin{array}{l}\text { Mínimos Cuadrados } \\
\text { Ordinarios }\end{array}$} & \multicolumn{4}{|c|}{ Error Espacial } \\
\hline & Coeficiente & Std. Error & & Coeficiente & Std. Error & \\
\hline Constante & 18,44395 & 0,0975 & $* * *$ & 18,4441 & 0,0942 & $* * *$ \\
\hline Habitaciones & $-0,04355$ & 0,0174 & $* *$ & $-0,04355$ & 0,0168 & $* *$ \\
\hline Edad & 0,00284 & 0,0042 & & 0,00284 & 0,0041 & \\
\hline Área construida & 0,00461 & 0,0004 & $* * *$ & 0,0046 & 0,0004 & $* * *$ \\
\hline Baños & 0,08971 & 0,0273 & $* *$ & 0,0897 & 0,0264 & $* * *$ \\
\hline Uso comercial & 0,22149 & 0,0737 & $* *$ & 0,22145 & 0,0712 & $* *$ \\
\hline Uso cultural & 0,20866 & 0,0839 & $* *$ & 0,20875 & 0,0812 & ** \\
\hline Uso educativo & $-0,1929$ & 0,0723 & $* *$ & $-0,19279$ & 0,0699 & * \\
\hline Uso industrial & 0,01798 & 0,0684 & & 0,01801 & 0,0661 & \\
\hline Uso servicios & 0,00512 & 0,0824 & & 0,00516 & 0,0796 & \\
\hline Zonas verdes y espacios abiertos & $-0,23196$ & 0,1088 & $* *$ & $-0,23186$ & 0,1051 & ** \\
\hline Centro del municipio & $-0,28907$ & 0,0804 & $* * *$ & $-0,28924$ & 0,0778 & $* * *$ \\
\hline Fragmentación & 0,91363 & 0,2335 & $* * *$ & 0,91338 & 0,2257 & $* * *$ \\
\hline R-cuadrado & \multicolumn{2}{|c|}{0,76} & & \multicolumn{2}{|c|}{0,76} & \\
\hline R-cuadrado ajustado & \multicolumn{2}{|c|}{0,7444} & & & & \\
\hline Error & \multicolumn{2}{|c|}{0,9881} & & & & \\
\hline Rezago & \multicolumn{2}{|c|}{0,8865} & & & & \\
\hline Robustez del error & \multicolumn{2}{|c|}{0,9859} & & & & \\
\hline Robustez del rezago & \multicolumn{2}{|c|}{0,8862} & & & & \\
\hline Coeficiente de error espacial & & & & 0,0033 & 0,1678 & \\
\hline
\end{tabular}

Los resultados sugieren que los precios de los inmuebles muestran que añadir un metro cuadrado en el área construida genera un aumento del 4,6\%; con la adición de un baño, el valor comercial incrementará en un 8,9\%. Posiblemente esto se debe a que la mayor parte de los predios presentan una alta cantidad de este tipo de dependencias y una incorporación de la misma no requiere una reducción significativa en el área construida.

Mosquera es un municipio que contempla la idea de localizar las áreas de actividad comercial y de servicios no solo en el centro del municipio sino en toda su extensión. Se estima que entre más aislada se encuentre la ubicación del predio de las zonas comerciales netas, el valor pedido por el inmueble tendrá un incremento del $22 \%$ por cada kilómetro que se aleje de dicha zona. Considerando que las zonas verdes y espacios abiertos son áreas que aportan al atractivo de un sector, se puede afirmar que la relación es positiva, en cuanto a la reducción de la distancia se refiere, es decir, el inmueble incrementará su valor en un $23 \%$ por cada kilómetro que se encuentre más cerca de este tipo de zonas. Como se predijo inicialmente la relación cuantitativa entre el centro del municipio y el valor de los predios es positiva, justificándose bajo la premisa que allí se encuentran ubicados los puntos más representativos y una alta proporción de las áreas residenciales con el mayor valor.

La variable en la que se hace énfasis de acuerdo a los propósitos del estudio es la medida de la 
fragmentación, siendo una de las más significativas. Los resultados de la variable se pueden evidenciar en la Figura 1, donde se presenta el mapa de la fragmentación espacial, la cual es dividida en quintiles, las áreas de uso residencial con comercio y servicios pertenecientes a la zona urbana son las más fragmentadas, representadas por los niveles más oscuros. Además, se evidencia un patrón en el que las vecindades menos fragmentadas se localizan en la zona rural, a excepción de la plataforma logística ALO en el segundo quintil, el suelo suburbano entre el segundo y tercer quintil y los centros poblados en el cuarto quintil. El patrón observado de la fragmentación espacial es consistente con estudios anteriores, como el de Kuethe [3] quien concluyó que los bajos niveles de fragmentación tienen un impacto negativo sobre el valor de los inmuebles, mientras que los altos producen un incremento. Por otro lado, Irwin \& Bockstael [29] encuentran que la diversidad crece y luego desciende a medida que se aleja del centro de la ciudad hacia la zona rural, esto se debe a que el incremento de la fragmentación se relaciona con la disminución de la superficie total de los parches, a un mayor número de fragmentos y una mayor dispersión de los mismos.

\section{CONCLUSIONES}

El valor comercial de los inmuebles varía directamente con la fragmentación espacial. Las posibles causas del comportamiento de esta variable son: un ineficiente plan de desarrollo urbanístico y la reducida intervención municipal en la gestión urbana,

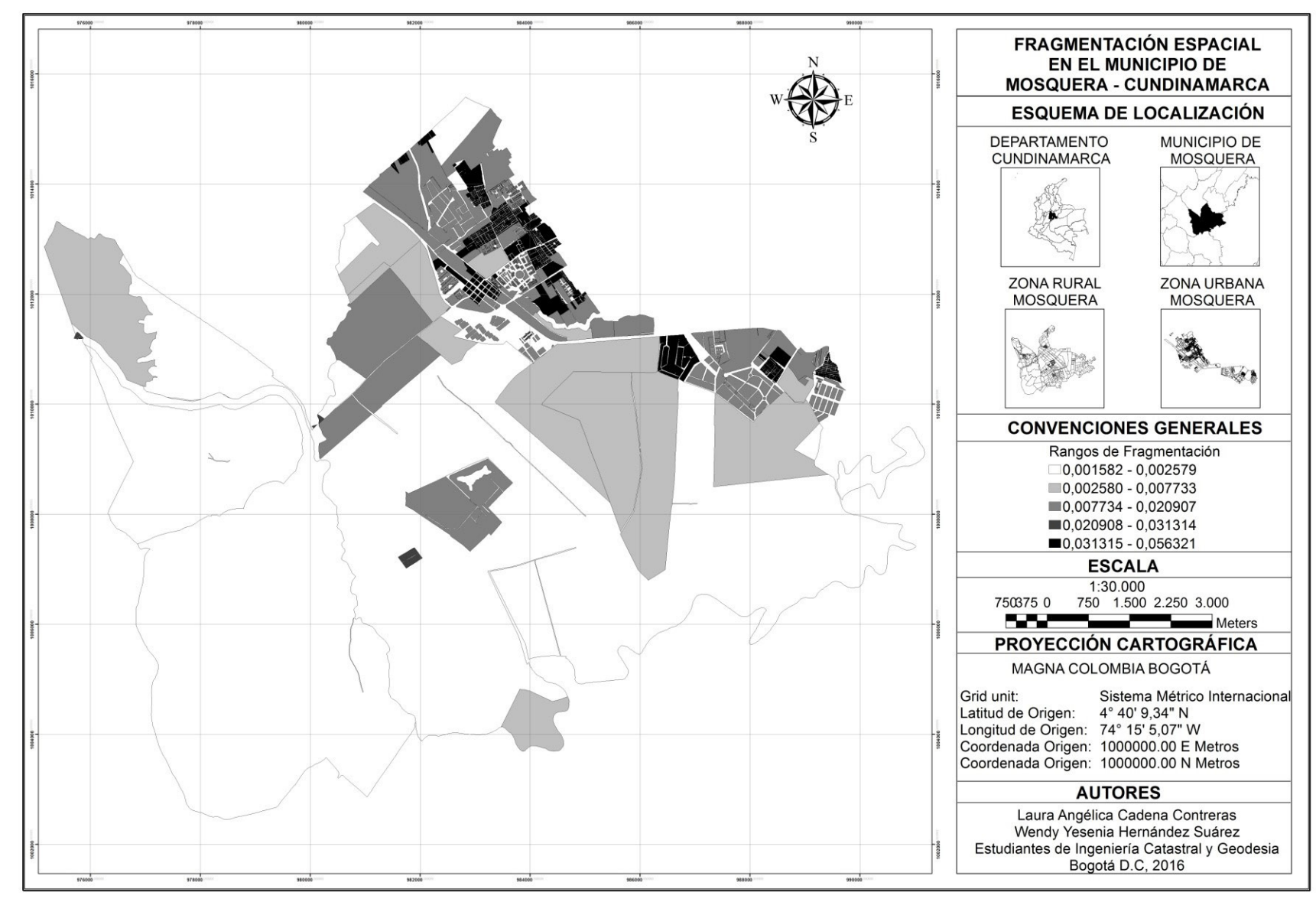

Figura 1. Mapa Fragmentación Espacial Mosquera, Cundinamarca. 
producidas por el apaciguamiento en las normas, el incremento de la dinámica inmobiliaria y la falta de integración del paisaje. Obteniendo como resultado un conjunto de fragmentos dispersos con diferentes usos de la tierra, sin ningún principio ordenador en común con la estructura urbana y regional. Por esta razón, estrategias de planificación urbana a corto plazo son las alternativas aconsejables para ordenar dichos fragmentos, así como propiciar la creación de escenarios adecuados para el desarrollo local y la expansión equilibrada del municipio.

El cálculo empírico de los precios implícitos y la estimación del valor de venta de la vivienda, obtenidos a partir del modelo hedónico, pueden extenderse tanto a los campos de la teoría urbana, como al análisis de las diferencias entre el precio de los inmuebles abordado desde la teoría monocéntrica o la multicéntrica, haciendo referencia esta última a la distancia a distintos centros de actividad económica. Además, si las entidades competentes gestionarán este tipo de investigación, se podría pensar en realizar comparaciones del precio de las transacciones inmobiliarias y de sus principales determinantes, tanto a nivel espacial como temporal.

El impacto de la expansión de los negocios inmobiliarios en el desarrollo urbano se hace evidente en la influencia que ha tenido Bogotá, D.C., sobre los municipios aledaños. Con el propósito de hacer la capital más competitiva frente a otras ciudades, busca atraer inversiones y movimiento de capital, materializando diferentes ofertas de proyectos, razón por la cual Mosquera se vio obligada a robustecer la reglamentación correspondiente a los usos de la tierra, su extensión y la diversidad de los mismos. Sin este tipo de limitaciones y de acuerdo a los resultados del estudio, una modificación en la superficie total de los parches, el número de fragmentos y la dispersión de los mismos, generaría un cambio en la fragmentación espacial y por consiguiente una variación significativa sobre el valor comercial de los bienes.

\section{REFERENCIAS}

[1] S. V. Koppmann, "segregación residencial y apropiación del espacio: la migración hacia las urbanizaciones cerradas del área metropolitana de Buenos Aires (Argentina)", Revista Electrónica de Geografía y Ciencias Sociales, no 94, pp. 1-11, 2001.

[2] E. Altinok y H. Cengiz, "The Effects of Urban Sprawl on Spatial Fragmentation and Social Segregation in Istanbul", Proceedings 44th ISOCARP Congress, 2008.

[3] T. H. Kuethe, "Spatial Fragmentation and the Value of Residential Housing", Land Economics, vol. 88, $\mathrm{n}^{\circ}$ 1, pp. 16-27, febrero 2012.

[4] H. Koster y J. Rouwendal, "The Impact of Mixed Land Use on Residential Property Values", Journal of Regional Science, vol. 52, pp. 733-761, 2012.

[5] S. Rosen, "Hedonic Prices and Implicit Markets: Produc Differentiations in Pure Competition", Journal of Political Economy, vol. 82, $\mathrm{n}^{\circ}$ 1, pp. 34-55, 1974.

[6] K. M. Kockelman, "The Effects of Location Elements on Home Purchase Prices and Rents in San Francisco Bay Area", Transportation Research Record: Journal of the Transportation Research Record, pp. 40-50, 1997.

[7] J. Geoghegan, L. A. Wainger y N. E. Bockstael, "Spatial Landscape Indices in a Hedonic Framework: An Ecological Economics Analysis Using GIS", Ecological Economics, vol. 23, n 3, pp. 251-264, 1997.

[8] M. A. Cruz, "Alcaldes de Cundinamarca, preocupados por el crecimiento urbano", 29 Febreo 2016. [En línea]. Consultado el 1 de marzo de 2016, disponible en http://www.eltiempo.com/ bogota/alcaldes-de-cundinamarca-preocupados-por-crecimiento-urbano/16524295?stopapp

[9] C. E. M. Martínez y Ó. O. M. Martínez, "Estimación de precios hedónicos para propiedades residencial y comercial en la ciudad de Bogotá", Ingeniería, vol. 8, no 1, pp. 10-17, 2003. 
[10] W. Alonso, Location and Land Use, Cambridge: Harvard University Press, 1964.

[11] D. P. McMillen, "Testing for Monocentricity", A Companion to Urban Economics, pp. 128140, 2006.

[12] L. L. S. Peña, "Alcances y límites de los métodos de análisis espacial", Papeles de Población, vol. 2, n 72, pp. 147-179, 2012.

[13] H. Arellano y J. O. Rangel, "Patterns in the distribution of vegetation in paramo areas: heterogeneity and spacial dependence", Caldasia, vol. 30, no 2, pp. 355-411, 2008.

[14] P. Aroca, "Econometría espacial: una herramienta para el análisis de la economía regional", Producción IDEAR, pp. 1-19, 2000.

[15] L. Anselin, "Spatial data analysis with GIS: an introduction to application", University of California, Santa Bárbara, 1992.

[16] L. Anselin2, "Under the hood Issues in the specification and interpretation of spatial regression models", Agricultural Economics, vol. 27, no 3, pp. 247-267, 2002.

[17] R. D. Baller, L. Anselin, S. F. Messner, G. Deane y D. F. Hawkins, "Structural covariates of U.S country homicide rates: Incorporating spatial effects", Criminology, vol. 39, no 3, pp. 561-589, 2001.

[18] L. Anselin3, "Econometric Theory", de Palgrave Handbook of Econometrics, vol. 1, $\mathrm{H}$. Hassani, T. Mills y K. Patterson, Edits., Palgrave Macmillan, 2006, pp. 901-941.

[19] J. A. P. Pineda, "Econometría espacial y ciencia regional", Investigación Económica, vol. 65, n² 258, pp. 129-160, Octubre 2006.

[20] J. M. Mueller y J. B. Loomis, "Spatial Dependence in Hedonic Property Models: Do Different Corrections for Spatial Dependence Result in Economically Significant Differences in Estimated Implicit Prices?", Journal of Agricultural and Resource Economics, vol. 33, ${ }^{\circ}$ 2, pp. 212-231, 2008.

[21] L. Anselin, A. K. Bera, R. Florax y M. J. Yoon, "Simple diagnostic test for spatial dependence", Regional Science \& Urban Economics, vol. 26, pp. 77-104, 1996.

[22] J. M. N. Tabales, N. C. Villamandos y G. M. Vázquez, "Aproximación a la valoración inmobiliaria mediante la metodología de precios hedónicos", de Conocimiento, innovación y emprendedores: camino al futuro, España, Universidad de la Rioja, 2007, pp. 2688-2701.

[23] S. Selim, "Determinants of house prices in Turkey: A hedonic regression model", Doğuş Üniversitesi Dergisi, vol. 9, no 1, pp. 65-76, 2008.

[24] S. Malpezzi, "Hedonic Pricing Models:A selective and Applied Review", Section in Housing Economics and Public Policy: Essays in Honor of Duncan Maclennan, pp. 1-43, 2002.

[25] H. J. F. Durán, "Desigualdad, segregación socio-espacial y precios del suelo en la ciudad latinoamericana. El caso de Bogotá en los años noventa", UIS Humanidades, vol. 38, n 1, pp. 109-126, 2010.

[26] Departamento Nacional de Planeación, " Planeación Nacional revela radiografía de gestión de 1.102 municipios", 23 de noviembre de 2015. [En línea]. Consultado el 2 de diciembre de 2015, disponible en https://www. dnp.gov.co/Paginas/Planeaci\%C3\%B3n-Nacional-revela-radiograf\%C3\%ADa-de-gesti\%C3\%B3n-de-1-102-municipios-.aspx

[27] Concejo Municipal de Mosquera, PBOT Mosquera, Mosquera, Cundinamarca, 2013.

[28] J. C. Martori y K. Hoberg, "Nuevas técnicas de estadística espacial para la detección de clusters residenciales de población inmigrante", Revista Electrónica De Geografía Y Ciencias Sociales, vol. 12, no 263, pp. 1-7, 15 Abril 2008.

[29] E. G. Irwin y N. E. Bockstael, "The evolution of urban sprawl: Evidence of spatial heterogeneity and increasing land fragmentation", Proceeding of the National Academy of Sciences of the United States of America, vol. 104, $\mathrm{n}^{\circ}$ 52, pp. 20672-20677, 2007.

[30] A. Ciocoletto, B. Gutiérrez y S. Ortiz, "Vivir en áreas residenciales monofuncionales desde 
la perspectiva de género", de Congreso Internacional de Vivienda Colectiva Sostenible, Barcelona, 2014.

[31] R. M. Serrano y E. V. Valcarce, "Econometría Espacial: Nuevas técnicas para el análisis regional. Una aplicación a las regiones europeas.", Investigaciones Regionales, vol. 1, pp. 83-106, 29 Junio 2004.

[32] S. Basu y T. G.Thibodeau, "Analysis of Spatial Autocorrelation in House Prices", Journal of Real Estate Finance and Economics, vol. 17, $\mathrm{n}^{\circ}$ 1, pp. 61-85, 1998.

[33] A. E. Gelfand, S. K. Ghosh, J. R. Knight y F. Sirmans, "Spatio-Temporal Modeling of Residential Sales Data", Journal of Business \& Economic Statistics, vol. 16, n 3, pp. 312-321, Julio 1998.

[34] F. Sánchez, A. M. Díaz y M. Formisano, "Conflicto, violencia y actividad criminal en CoIombia: Un análisis espacial", Documento CEDE, pp. 1-60, 2003.
[35] M. Formisano, "Econometría Espacial: Características de la violencia homicida en Bogotá.", Documentos CEDE, pp. 1-42, Septiembre 2002.

[36] E. V. Marsal, "La concentración territorial de las empresas industriales: un estudio sobre la unidad geográfica de análisis mediante técnicas de econometría espacial", Documents de treball IED, pp. 1-26, 2001.

[37] W. Tobler, "On the First Law of Geography: A Reply", Annals of the Association of American Geographers, vol. 94, no 2, pp. 304-310, 2004.

[38] Alcaldía Municipal de Mosquera, "Sitio Oficial de Mosquera", 2016. [En línea]. Consultado el 2 de diciembre de 2015, disponible en http:// mosquera-cundinamarca.gov.co/index.shtml\#4

[39] Centre for Northern Forest Ecosystem Research (Ontario Ministry of Natural Resources), "Patch Analyst For ArcGIS®", 2012. [En línea]. Consultado el 4 de marzo de 2016, disponible en http://flash.lakeheadu.ca/ rrempel/patch/images/patchanalyst.pdf 